\title{
Working Memory for Meaningless Manual Gestures
}

\author{
Mary Rudner
}

\section{Linköping University Post Print}

\section{Tweet}

N.B.: When citing this work, cite the original article.

Original Publication:

Mary Rudner, Working Memory for Meaningless Manual Gestures, 2015, Canadian journal of experimental psychology, (69), 1, 72-79.

http://dx.doi.org/10.1037/cep0000033

Copyright: Canadian Psychological Association

http://www.cpa.ca/

Postprint available at: Linköping University Electronic Press

http://urn.kb.se/resolve?urn=urn:nbn:se:liu:diva-116820 
WM for meaningless manual gestures

Working memory for meaningless manual gestures

Mary Rudner

Linnaeus Centre HEAD, Department of Behavioural Sciences and Learning, Linköping University, Sweden.

Adress for correspondence:

Mary Rudner, PhD

Department of Behavioural Sciences and Learning

Linköping University

58183 Linköping

Sweden

Email:mary.rudner@liu.se

Telephone: +4613282157

Fax: +4613282145 
Working memory for manual gestures

Effects on working memory performance relating to item similarity have been linked to prior categorization of representations in long-term memory. However, there is evidence from gesture processing that this link may not be obligatory. The present study investigated whether working memory for incidentally generated meaningless manual gestures is influenced by formational similarity and whether this effect is modulated by working memory load. Results showed that formational similarity did lower performance, demonstrating that similarity effects are not dependent on prior categorization. However, this effect was only found when working memory load was low, supporting a flexible resource allocation model according to which it is the quality rather than quantity of working memory representations that determines performance. This interpretation is in line with proposals suggesting language modality specific allocation of resources in working memory.

Keywords: working memory; sign language; manual gestures; load; flexible resource allocation; 
Working memory for manual gestures

Working memory is the capacity to maintain and process information on-line (Baddeley, 2000). This capacity is generally considered to be limited to three to four items (Cowan, 2001), but is influenced by the availability of associated linguistic representations in long-term memory. Thus, working memory capacity for verbal items or items that can be named is greater than for shapes that are hard to categorize. However, if the verbal items share phonological features, they may be harder to remember than items that are phonologically distinct and it has been suggested that this is due to confusion among representations held in memory (Baddeley, 2000). The phonological similarity effect generalizes to the gesture-based natural sign languages used by deaf communities (Andin et al., 2013; Wilson \& Emmorey, 1997). Sign languages have a sublexical structure which sets them aside from gesture systems and that can be understood in terms of contrast in the shape, location and movement of the signing hands (Emmorey, 2002). However, reduced working memory capacity for manual gestures with formational similarity has also been found for unfamiliar, nonmeaningful body movements (Wilson \& Fox, 2007), suggesting that the phonological similarity effect found for sign language may not be entirely dependent on the existence of linguistic representations in long term memory. However, the stimuli used by Wilson and Fox in their 2007 study consisted of gestures based on combinations of handshapes, locations, and movements, following the phonological structure of sign language. Thus, although the stimuli were unfamiliar and meaningless to the signnaïve hearing participants, they were clearly generated in an intentional manner and thus open to the categorization that may drive the linguistic similarity effects that influence working memory performance. In the present study, it was investigated whether formational similarity influences working memory processing of non-linguistic manual gestures that are generated incidentally without prior categorization. Were this to be the case, it would allow the possibility that similarity effects in sign language may be to some extent driven by visual similarity and not just by the similarity of cognitive representations.

The gestures used as stimuli in the present study were generated in the following manner: a seated model was video-recorded catching a ball thrown by an accomplice standing close to the video 
Working memory for manual gestures

camera towards a range of locations proximal to the model. After recording, these stimuli were categorized according to the quadrant of space in which the model caught the ball. Thus, manual gestures were achieved that were generated incidentally and which could be subsequently categorized according their spatial characteristics. It can be confidently assumed that such items do not have specific representations in long-term memory.

Although there is consensus that working memory capacity is limited to a handful of items when language-based strategies are overruled, there is controversy concerning the nature of that limitation (Fukuda, Awh \& Vogel, 2010). One view is that working memory capacity is defined by a limited number of discrete slots that can accommodate one item each, irrespective of the complexity of that item (Luck \& Vogel, 1997). The opposing view is that working memory capacity constitutes a flexible resource that can be assigned without a set limit (Bays \& Husain, 2008). According to the slots model, item similarity should not affect working memory capacity when load does not challenge capacity because there will be sufficient slots for all items irrespective of their features. However, when load is beyond capacity, item similarity will compromise performance. According to the flexible resource model, however, resources are allocated flexibly in relation to item complexity and task demands. In other words, the flexible resource model proposes that it is the quality, rather than the quantity, of working memory representations that determines performance (Ma, Husain \& Bays, 2014). Although the flexible resource model was developed to explain phenomena in the visuospatial domain, it is in tune with the Ease of Language Understanding (ELU) model of working memory for language processing (Rönnberg et al., 2013) which proposes that ease of language understanding is determined by the smooth functioning of an episodic buffer (Baddeley, 2000; Rudner \& Rönnberg, 2008) which depends on the quality of language input and the quality of corresponding representations in long-term memory. Recent work suggests that the function of the episodic buffer is also dependent on other task demands (Mishra, Stenfelt, Lunner, Rönnberg \& Rudner, 2014). 
Working memory for manual gestures

Recall of to-be-remembered items is a useful approach to measuring working memory capacity when corresponding representations are distinctly categorized in long-term memory, for example as entries in the mental lexicon (Baddeley, 2000). One example is the reading span task (Daneman \& Carpenter, 1980; Rönnberg, Arlinger, Lyxell \& Kinnefors, 1989) which taps parallel storage and processing capacity by requiring participants to read and semantically process sentences while at the same time retaining their content in memory. However, when to-be-remembered items have no existing representations in long-term memory, another approach is needed; one candidate is the nback task (Cohen, Forman, Braver, Casey, Servan-Schreiber, Noll, 1994; Dahlin, Stigsdotter Neely, Larsson, Bäckman \& Nyberg, 2008).

In an n-back paradigm, series of items are presented and the task is to match each item to the item that preceded it $\mathrm{n}$ steps back in the series. Thus, the participant determines whether a yes or no response is appropriate rather than having to reproduce specific items. This task requires the temporary maintenance and processing of items that constitute the hallmark of working memory tasks (Baddeley, 2000). It has been shown to reliably activate the frontoparietal network that characterizes working memory, demonstrating its validity as a working memory task (Owen, McMillan, Laird \& Bullmore, 2005). Further, the n-back task has been used for both shapes and spatial arrays (Smith and Jonides, 1997) as well as for lexical signs (Rudner, Fransson, Nyberg, Ingvar \& Rönnberg, 2007) and both sign- and speech-based phonological recoding of pictures (Rudner, Karlsson, Gunnarsson \& Rönnberg, 2013). Because working memory capacity is limited, when maintenance costs increase, for example when more items need to be stored in working memory generating greater load, performance decreases (Owen et al., 2005; Smith and Jonides, 1997). N-back paradigms manipulating memory load allow simultaneous orthogonal manipulation of other factors such as task difficulty (Barch, Braver, Nystrom, Forman, Noll \& Cohen, 1997). Thus, the n-back paradigm can be used for manipulating load and other factors of interest simultaneously to identify potential interactions. 
Working memory for manual gestures

It has been demonstrated that $\mathrm{n}$-back performance is decreased by phonological similarity among the verbal labels of stimuli that consist of printed consonants (Sweet et al., 2008). However, the functional magnetic resonance imaging study by Sweet et al (2008) showed that phonological similarity was associated with deactivation of cerebral regions associated with attention and language functions rather than modulation of activation in the core fronto-parietal working memory network. This finding was interpreted to suggest that attention and language functions may disengage in the face of distracting information. This might in turn lead to reduced richness of representation, in line with a resource allocation view of working memory (Ma et al., 2014).

The aim of the present study was to investigate whether working memory for meaningless manual gestures is influenced by the formational similarity of those gestures and thus whether there is a basis for entertaining the possibility that the phonological similarity effect identified for sign language may be attributable to non-linguistic processing. To achieve this, an n-back working memory task based on incidentally generated meaningless manual gestures was administered to hearing non-signers. Non-signers were used to avoid any processing contamination from bias towards visual processing engendered by auditory deprivation and experience of using manual gestures linguistically (Bavelier, Dye \& Hauser, 2006). Two memory load levels were used (1-back and 2-back) and the spatial proximity of the non-target items to the target items was manipulated. It was predicted that performance would decrease with increasing memory load as found in previous nback studies and that the presence of spatially proximal non-target items would interfere with performance, indicating an effect of formational similarity that could not be explained with reference to representations stored in long-term memory. An interaction between load and proximity was also predicted whereby a greater effect of proximity with greater load was expected. An effect of proximity at low load would provide support for the flexible resource allocation model while a lack of such an effect would support the slot model. 
Working memory for manual gestures

Methods

Participants

20 undergraduates (13 women) at Linköping University were recruited to the study. The mean age was $25(S D=2)$. The study was approved by the regional ethical review board in Linköping and all participants gave informed written consent.

$\mathrm{N}$-back task

The stimuli used in the $n$-back task were video-recordings of a female model catching a small ball thrown towards her by an accomplice who stood behind the video camera. The accomplice threw the ball towards a range of locations proximal to the body of the model during continuous video recording. After recording, four-second catching sequences were clipped from the continuous recording and categorized according to the quadrant of space (upper left, upper right, lower left, lower right) in which the ball was caught. The ball-catching stimuli were arranged in eight sequences of forty items such that there were 15 evenly but not regularly dispersed targets per condition. In half the sequences, 15 of the foils (non-target items) came from the same quadrant of space as the adjacent targets and thus constituted proximal foils while in the other half the foils always came from a different quadrant of space and thus constituted distal foils, see Figure 1 for examples. There were two different sequences for each condition to control for material effects. The participants were instructed to determine for each item whether it exactly matched the nth back item in the series. For the 1-back task this meant determining whether each item exactly matched the immediately preceding item and for the 2-back task this meant determining whether each item exactly matched the preceding item but one, see Figure 1 for examples.

Insert Figure 1 about here.

Thus, the task had a $2 \times 2$ within group experimental design with the factors load $(n=1, n=2)$ and foil proximity (yes, no). Each participant performed the task once in each of the four conditions. The two 
Working memory for manual gestures

$\mathrm{n}=1$ conditions were performed consecutively and preceded by a practice list, as were the two $n=2$ conditions. The order of presentation of load was balanced across participants as was the order of foil proximity at each load. Stimuli were delivered and yes/no button-press responses were captured using DMDX software (Forster \& Forster, 2003). The interval between stimulus onsets was 5000 ms.

\section{Reading span}

This test requires maintenance and processing of visually presented printed sentences and is widely used as a measure of individual working memory capacity (Daneman \& Carpenter, 1980; Mishra, Lunner, Stenfelt, Rönnberg \& Rudner, 2013; Rönnberg et al. 1989). In particular, because the reading span task requires storage and processing of lexical items that need to be retrieved from semantic long-term memory, it taps into verbal processes and is thus specifically a measure of verbal working memory and as such is a robust predictor of performance on challenging language tasks (Just \& Carpenter, 1992). In the version of the reading span task used in the present study (Mishra et al., 2013), 54 three-word sentences were presented word by word on the computer screen. Each word was visible for $800 \mathrm{~ms}$ with a $50 \mathrm{~ms}$ interval between words. These sentences were presented in sets ranging in length from two to six sentences, in ascending order. There were three sets of each length. Half of the sentences were coherent and half were nonsense. After each sentence, the participant had 1.75 seconds to decide whether it made sense or not. After each set of sentences, the participant was prompted to recall either the first or the last word of all the sentences in the set, in the order of presentation. Post cuing of items for recall is a common feature of recent versions of the reading span task (e.g. Mishra et al., 2013) which serves to combat selective encoding thus increasing working memory load. The dependent variable was the total number of words correctly recalled in any order.

Ball sense rating

Because the $n$-back task involved processing visual representations of the motor actions generated when catching a ball, it was reasoned that individual differences in the ability to perform such 
Working memory for manual gestures

processing may have an influence on $n$-back task performance. It was further reasoned that the participants were likely to have a reasonably reliable opinion of their own ability in this regard and that regular practice in a ball sport would be likely to enhance such ability. Thus, participants were asked to rate their ball sense on a Likert scale of one to seven where one is poor and seven is excellent. They were also asked if they played any ball sport regularly and if so which one.

Procedure

The participants were informed about the study and provided their written consent. They then provided demographic data, rated their ball sense and performed the $n$-back experiment. After the $\mathrm{n}$-back experiment they were debriefed about the strategies they used for solving the $\mathrm{n}$-back task. Finally, the participants performed the reading span task.

Results

Target accuracy. There were 15 target trials per condition and thus maximum possible target accuracy was 15. Mean accuracy in each condition is shown in Table 1. Repeated measures ANOVA showed a significant main effect of $N, F(1,19)=31.81, \mathrm{MSE}=1.56, p=0.000$, partial $\eta 2=0.63$, such that accuracy was greater for 1-back than for 2-back, but no significant main effect of Proximity, $F(1$, $19)=1.82, \mathrm{MSE}=1.55, p=0.19$ and only a tendency towards a significant interaction, $F(1,19)=4.13$, MSE $=.88, p=.056$. However, because we had predicted an interaction we tested the simple main effects. There was a significant difference between 1-back and 2-back performance both with and without proximal foils (ps $<0.001$ ). There was no significant difference between performance with and without proximal foils at $n=1(p=.868)$. However, at $n=2$, there was a significant difference, $F(1,19)=7.31, p=.014$, with worse target accuracy when there were no proximal foils. It is possible that the presence of proximal foils encouraged the participants to make more positive matching judgments when load was high. Thus, the ability to identify targets was high overall, influenced by working memory load and inflated by task strategy when working memory load was high. Therefore it is important to take errors into account when assessing performance. 
Working memory for manual gestures

Insert Table 1 about here.

Errors. It was possible to make errors on both targets and foils and thus the maximum number of errors per condition was 40. Mean errors in each condition are shown in Table 1. Repeated measures ANOVA showed two significant main effects and a significant interaction. The main effect of $\mathrm{N}$, $F(1,19)=19.7, \operatorname{MSE}=7.7, p=0.000$, partial $\eta 2=.51$, showed that more errors were made for 2-back than 1-back; the main effect of proximity, $F(1,19)=13.48, \mathrm{MSE}=6.23, p=0.002$, partial $\eta 2=0.42$, showed that more errors were made when foils were proximal rather than distal; the interaction, $F(1,19)=32.52, \mathrm{MSE}=3.54, p=0.000$, partial $\eta 2=0.63$, showed that foil proximity had a greater effect for 1-back than 2-back.

D'. Because target accuracy was high across conditions and error rate varied across conditions we calculated d' (Stanislaw \& Todorov, 1999) to adjust hits (correct performance on target trials) for false alarms (incorrect performance on non-target trials). The maximum possible d' score was 2.84 (no errors). Mean d' by condition is shown in Table 1. Repeated measures ANOVA showed two significant main effects and a significant interaction. The main effect of $N, F(1,19)=33.67$, MSE $=.16$, $p=0.000$, partial $\eta 2=.64$, showed that $d^{\prime}$ was greater for 1-back than 2-back; the main effect of proximity, $F(1,19)=10.69$, MSE $=.13, p=0.004$, partial $\eta 2=.36$, showed that $d^{\prime}$ was greater with distal than proximal foils; the interaction, $F(1,19)=36.49, \mathrm{MSE}=.07, \mathrm{p}=0.000$, partial $\eta 2=.66$, showed that the effect of proximity was greater for 1-back than 2-back, see Figure 2 . Thus, taking d' prime as an adjusted measure of performance, it is shown that foil proximity results in lower working memory performance when load is low $(n=1)$ but not when it increases $(n=2)$.

Insert Figure 2 about here.

Latency. Mean latency, irrespective of trial type and accuracy, is shown in Table 1. Repeated measures ANOVA showed two significant main effects and a significant interaction. The main effect of $\mathrm{N}, \mathrm{F}(1,19)=63.03, \mathrm{MSE}=30562.15, \mathrm{p}=0.000$, partial $\eta 2=.77$, showed slower performance for 2 back than for 1-back; the main effect of proximity, $F(1,19)=18.69, \mathrm{MSE}=35402.46, p=0.000$, 
Working memory for manual gestures

partial $\eta 2=.50$, showed slower performance with proximal than distal foils; the interaction, $F(1,19)$ $=17.12, \mathrm{MSE}=24430.97, \mathrm{p}=0.000$, partial $\eta 2=.47$, showed that the effect of proximity was greater for 1-back than 2-back. Thus, the patterns of effects obtained for latency, mirrors the pattern obtained for $d^{\prime}$. Together, the pattern of results from the $n$-back experiment demonstrates that during an n-back working memory task based on meaningless manual gestures, the presence of proximal foils reduces performance. The effect of proximal foils differs with load. When working memory load is low, proximal foils slow performance and generate more errors but do not influence hits. When working memory is high, proximal foils generate more hits and errors, which cancel each other out.

Strategy. Nine out of the twenty participants stated that they used a visual strategy only, while 11 stated that they used a verbal or mixed strategy. When strategy was entered into the d'ANOVA as a between group factor there was no significant main effect of strategy, $F(1,18)=.001$, MSE $=.20, p=$ .98 , partial $\eta 2=.00$, and there was no significant interaction with either of the within group factors, ps $>.16$. Thus, there was no evidence that the conscious strategies the participants used to solve the task had any influence on performance.

\section{Reading span}

A total of 54 sentences are presented in the reading span task, giving a maximum total score on the recall and semantic judgment parts of the tasks of 54 . Mean performance on the recall part was $27.10(S D=5.50)$, which is in line with previous findings (e.g. Mishra et al., 2013) but mean performance on the semantic judgment part of the test was only $26.95(S D=2.76)$ which is relatively low compared to previous studies which have found near ceiling performance for this component (e.g. Mishra et al., 2013). The relatively low score in the present study suggests that the participants were trading off cognitive resources across the two parts of the dual task. The two scores were not significantly correlated, Spearman's rho $=.23, p=.16$.

Ball sense 
Working memory for manual gestures

Participants rated their ball sense on a Likert scale 1-7 where 1 indicated poor ball sense and 7 indicated excellent ball sense. This provides a measure of the ability to process representations of the motor actions generated when catching a ball. Mean rating was $4.5(S D=1.32)$. Three participants stated that they played a ball sport regularly, which was football in every case. There was no difference in rated ball sense between those who played football regularly and those who did not, $t(18)=.23, p=.82$ and ball sense did not correlate with reading span recall, Spearman's rho $=-.16$, $p=.25$ or semantic judgment, Spearman's rho $=-.002, p=.50$. Thus, there was no evidence that the ability to process representations of the motor actions generated when catching a ball was associated with verbal working memory.

\section{Correlations}

Spearman's correlations were computed between the $d^{\prime}$ measure of $n$-back performance and reading span as well as rated ball-sense, see Table 2 . Working memory performance measured using the reading span task did not correlate significantly with n-back performance. Further, it should be noted that all the coefficients of the correlations between reading span and the $d^{\prime}$ measures are negative and thus that any tendency to an association discernable among these correlations would suggest that better reading span performance is associated with poorer ability to solve the $n$-back task. This may explain why verbal strategies did not enhance performance. Rated ball sense, on the other hand, correlated significantly and positively with n-back performance overall and specifically in 1-back conditions as well as when foils were proximal, suggesting that having good ability to process the representations of the motor actions generated when catching a ball may actually have helped participants to perform better on the n-back task, particularly when targets were perceptually hard to distinguish from foils and when working memory load was low.

Insert Table 2 about here. 
Working memory for manual gestures

\section{Discussion}

The results of the present study clearly show that non-linguistic manual gestures in the form of ballcatching events can be successfully stored and processed in working memory and that performance decreases with increasing memory load as predicted. It was also found that the presence of spatially proximal non-target items interfered with performance. This effect cannot be explained with reference to representations stored in long-term memory. Thus, it demonstrates that formational similarity of manual gestures interferes with working memory capacity even when they cannot be categorized on the basis of existing linguistic representations in long term memory. This confirms previous work on categorized gestures (Wilson \& Fox, 2007) and extends it to manual gestures that are generated incidentally without prior categorization. It also opens up the possibility that similarity effects in sign language may be driven by visual similarity and not just by similarity of cognitive representations.

Although an interaction was found between working memory load and foil proximity, its nature was not as predicted. Instead of the expected greater effect of foil proximity with greater load, proximity only influenced the $d^{\prime}$ measure of performance when memory load was low. I argued that the nature of the interaction between load and proximity would be able to help adjudicate between the competing accounts of working memory capacity: the slot model (Fukuda et al., 2010) and the flexible resource model (Ma et al., 2014). In particular, I argued that an effect of proximity at low load would provide support for the flexible resource allocation model while the lack of such an effect would support the slot model. In order to solve the 1-back task only two items have to be held in working memory: the current item and the previous item. This is well below the proposed limit for working memory of three to four items and thus constitutes a low-load condition. Because there was a clear effect of proximity on the $d^{\prime}$ measure at low load $(n=1)$, support was found for the flexible resource model. 
Working memory for manual gestures

The effect of proximity at low load was not accompanied by a greater effect of proximity on the d' measure at $n=2$, which we have referred to as high load. In order to solve the 2-back task, three items need to be held in working memory: the current item, the previous item and the previous item but one. This is close to the limits of working memory for non-verbal information. Indeed, if there is any inefficiency in working memory processing at $n=2$ due to imperfect updating, more than three items may be maintained in working memory at any one time, critically overloading the system. Thus, it is likely that in the present study all available resources were allocated irrespective of proximity at $n=2$, reflecting task difficulty.

Interestingly, there was an effect of foil proximity on target accuracy at $n=2$ such that more targets were accurately identified when proximal foils were present. The fact that this effect was cancelled out in the d' measure suggests that there was a response bias when working memory load was high. It suggests that at $n=2$, items were sufficiently richly encoded to determine that the current item and the last item but one shared a spatial location but not to reliably determine whether they were visually similar or not, leading to a bias towards accepting co-located pairs. This interpretation further supports a flexible resource allocation interpretation of the results.

Rated ball sense predicted performance at $n=1$ but not at $n=2$. Further, rated ball sense predicted performance with proximal foils but not distal foils. These associations suggest that working memory processing of meaningless gestures at low memory load and coping with visuospatial confusion during the $n$-back task were supported by visual skills, providing evidence that these tasks tap into low-level visual processing mechanisms. Importantly, there was no significant correlation between reading span performance which was our measure of verbal working memory and n-back performance. Thus, there is no evidence of shared mechanisms for verbal working memory and working memory for meaningless gestures in hearing non-signers. Indeed it should be noted that the coefficients of all the correlations between $n$-back performance and reading span were negative, suggesting that good verbal working memory skills may even have interfered to some extent with 
Working memory for manual gestures

working memory for meaningless manual gestures. This provides a possible explanation for the lack of association between verbal strategies and performance. Inclusion of an established test of visuospatial working memory would have allowed examination of an association with the $n$-back task and potentially provided stronger evidence of the underlying construct.

Inspection of Figure 2 shows that performance on the 2-back task was similar to performance on the 1-back task when proximal foils were present. Thus, the effect of increasing memory load was similar to the effect of introducing proximal foils. In other words, it was just as hard to reject an item as a non-match when it was visuospatially similar to the target as when there was an intervening representation to be maintained in working memory. Thus, with the 2-back task, the limits of working memory may have been breached. This interpretation is also in line with the resource allocation model of working memory.

We perceive the visual world to be highly detailed but it is an illusion that the full richness of detail is available to cognitive processing (Simons \& Rensink, 2005) It has been suggested that one of the functions of working memory is to maintain that visual illusion by bridging the temporal gaps between fixations and the spatial gaps between saccades that iconic memory does not cope with (Luck \& Vogel, 2013). Although working memory engages an extensive frontoparietal network (Owen et al., 2005; Smith \& Jonides, 1997), it has been demonstrated that a single bilaterally symmetric area in the intraparietal and intraoccipital sulci is the locus of a capacity-limited store for the representation of the visual scene (Todd \& Marois, 2004).

Cognizing the visual scene is vital for sign language communication. A body of work has investigated working memory for sign language and found that although it is functionally similar to working memory for speech and supported by similar neural mechanisms, there are language modality specific differences in the neural networks that support working memory (for a review see Rudner, Andin \& Rönnberg, 2009). In particular, working memory for sign language specifically recruits bilateral superior parietal regions associated with visuospatial processing (Bavelier, Newman, 
Working memory for manual gestures

Mukherjerr, Hauser, Kemeny, Braun \& Boutla, 2008; Rönnberg, Rudner \& Ingvar, 2004; Rudner, Fransson, Nyberg, Ingvar \& Rönnberg, 2007). This suggests that there are fundamental differences in the working memory processing of oral and gesture-based languages, despite considerable functional similarities (Rönnberg et al., 2013; Rudner et al., 2009; Rudner \& Rönnberg, 2008). It is likely that these differences are related to sensorimotor mechanisms supporting the two language modalities and the ways in which they are represented (Emmorey, McCullough, Mehta \& Grabowski, 2014). However it has also been suggested that modality specific activation of parietal cortex reflects language modality specific reliance on executive strategies during working memory tasks (Bavelier et al., 2008).

It is interesting to note that the bilateral superior parietal regions that characterize working memory for sign language compared to speech are adjacent to and possibly overlapping with the capacitylimited store for the representation of the visual scene identified by Todd and Marois (2004). One possibility is that the bilateral superior parietal activation found for working memory for sign language compared to speech at least partially reflects processing of the formational features of gestures in the capacity-limited store for the representation of the visual scene. This notion is in line with previous proposals that this activation reflects generation of a virtual spatial array (Rönnberg et al., 2004; Rudner et al., 2007) supporting order processing (Rudner et al., 2009).

Finally, because sign language users have representations of lexicalized gestures stored in the mental lexicon in long-term memory, working memory capacity for familiar signs is likely to be greater in this group than for meaningless manual gestures. Thus, while either engagement of the capacity-limited store for the representation of the visual scene may explain modality-specific processing in working memory for sign language, amodal networks are likely to reflect maintenance and processing of existing representations.

Conclusion 
Working memory for manual gestures

The results of the present study show that working memory capacity for incidentally generated meaningless manual gestures is reduced when those gestures are formationally similar, although there are no corresponding representations in long-term memory. This opens up the possibility that similarity effects found previously in gesture-based working memory tasks may be due at least in part to visual similarity and not only to similarity among cognitive representations. It is tentatively suggested that previous findings of sign-language specific activation of the bilateral superior parietal lobes may reflect activation of a capacity-limited store for the representation of the visual scene. 
Working memory for manual gestures

\section{Acknowledgement}

Thanks to Åsa Christiansson for help with data collection. This work was supported by Riksbankens jubileumsfond grant number P2008-0481:1-E to the author. 
Working memory for manual gestures

\section{References}

Andin, J., Orfanidou, E., Cardin, V., Holmer, .E, Capek, C.M., Woll, B., Rönnberg, J. and Rudner, M. (2013). Similar digit-based working memory in deaf signers and hearing non-signers despite digit span differences. Frontiers in Psychology, 4, 942. doi: 10.3389/fpsyg.2013.00942

Baddeley, A. (2000). The episodic buffer: A new component of working memory? Trends in Cognitive Sciences, 4, 417-423.

Barch, D.M., Braver, T.S., Nystrom, L.E., Forman, S.D., Noll, D.C. \& Cohen, J.D. (1997). Dissociating working memory from task difficulty in human prefrontal cortex. Neuropsychologia, 35, 1373-1380.

Bavelier, D., Dye, M.W.G. \& Hauser, P.C. (2006). Do deaf individuals see better? TRENDS in Cognitive Sciences, 10(11), 512-518

Bavelier, D., Newman, A.J., Mukherjerr, M., Hauser, P., Kemeny, S., Braun, A. \& Boutla, M. (2008). Encoding, rehearsal, and recall in signers and speakers: shared network but differential engagement. Cerebral Cortex, 18, 2263-2274.

Bays, P.M. \& Husain, M. (2008). Dynamic shifts of limited working memory resources in human vision. Science, 321, 851-854.

Braver, T. S., Cohen, J. D., Nystrom, L. E., Jonides, J., Smith, E. E., \& Noll, D. C. (1997). A parametric study of prefrontal cortex involvement in human working memory. Neuroimage, 5, 49-62.

Cohen, J.D., Forman, S.D., Braver, T.S., Casey, B.J., Servan-Schreiber, D., Noll, D.C. (1994). Activation of the prefrontal cortex in a nonspatial working memory task with functional MRI. Human Brain Mapping, 1(4), 293-304. doi: 10.1002/hbm.460010407.

Cowan, N. (2001). The magical number 4 in short-term memory: A reconsideration of mental storage capacity. Behavioral and Brain Sciences, 24, 87-185. 
Working memory for manual gestures

Dahlin, E., Stigsdotter Neely, A., Larsson, A., Bäckman, L. \& Nyberg, L. (2008). Transfer of Learning After Updating Training Mediated by the Striatum. Science 320, 1510, DOI: 10.1126/science.1155466

Daneman,M. \& Carpenter,P.A. (1980). Individual differences in integrating information between and within sentences. Journal of Experimental Psychology: Learning Memory and Cognition, 9, 561-584.

Emmorey, K., McCullough, S., Mehta, S. \& Grabowski,T.J. (2014). How sensory-motor systems impact the neural organization for language: direct contrasts between spoken and signed language. Frontiers in Psychology, 5, 484. doi: 10.3389/fpsyg.2014.00484

Forster, K. I., \& Forster, J. C. (2003). DMDX: A windows display program with millisecond accuracy. Behavior Research Methods, Instruments, \& Computers, 35, 116-124. doi: 10.3758/BF03195503

Fukuda, K., Awh, E. \& Vogel, E.K. (2010). Discrete capacity limits in visual working memory. Current Opinion in Neurobiology, 20, 177-182.

Just, M.A. \& Carpenter, P.A. (1992). A capacity theory of comprehension: individual differences in working memory. Psychological Review, 99(1), 122-149.

Luck, S.J. \& Vogel, E.K. (1997) The capacity of visual working memory for features and conjunctions. Nature 390, 279-281.

Luck, S.J. \& Vogel, E.K. (2013). Visual working memory capacity: from psychophysics and neurobiology to individual differences. Trends in Cognitive Sciences,17(8), 391-400.

Ma, W.J., Husain, M. \& Bays, P.M. (2014). Changing concepts of working memory. Nature Neuroscience, 17(3), 347-356.

Mishra, S., Lunner, T., Stenfelt, S., Rönnberg, J. \& Rudner, M. (2013). Seeing the talker's face supports executive processing of speech in steady state noise. Frontiers in Systems Neuroscience, 7:96. doi: 10.3389/fnsys.2013.00096 
Working memory for manual gestures

Mishra, S., Stenfelt, S., Lunner, T., Rönnberg, J. \& Rudner, M. (2014). Cognitive spare capacity in older adults with hearing loss. Frontiers in Aging Neuroscience, 6, 96. doi: 10.3389/fnagi.2014.00096

Owen, A.M., McMillan, K.M., Laird, A.R. \& Bullmore, E., (2005). N-Back Working Memory Paradigm: A Meta-Analysis of Normative Functional Neuroimaging Studies. Human Brain Mapping, 25, 46 -59.

Rönnberg, J., Arlinger, S.,Lyxell, B., \& Kinnefors, C.(1989). Visual evoked potentials: Relation to adult speechreading and cognitive function. Journal of Speech, Language, and Hearing Research, 32, 725735.

Rönnberg, J., Lunner, T., Zekveld, A.A., Sörqvist, P., Danielsson, H., Lyxell, B., Dahlström, Ö., Signoret, C., Stenfelt, S., Pichora-Fuller, M.K. \& Rudner, M. (2013). The Ease of Language Understanding (ELU) model: Theoretical, empirical, and clinical advances. Frontiers in Systems Neuroscience, 7, 31. doi: 10.3389/fnsys. 2013.00031

Rönnberg, J., Rudner, M. \& Ingvar, M. (2004). Neural Correlates of Working Memory for Sign Language. Cognitive Brain Research, 20, 165-182.

Rudner, M., Andin, J. \& Rönnberg, J. (2009). Working memory, deafness and sign language. Scandinavian Journal of Psychology, 50, 495-505.

Rudner, M., Fransson, P., Ingvar, M., Nyberg, L. \& Rönnberg, J. (2007). Neural representation of binding lexical signs and words in the episodic buffer of working memory. Neuropsychologia, 45(10), 2258-2276.

Rudner, M., Karlsson, T., Gunnarsson, J. \& Rönnberg, J. (2013). Levels of processing and language modality specificity in working memory. Neuropsychologia, 51, 656-666. DOI: 10.1016/j.neuropsychologia.2012.12.011.

Rudner, M. \& Rönnberg, J. (2008). The role of the episodic buffer in working memory for language processing. Cognitive Processing, 9, 19-28. doi 10.1007/s10339-007-0183-x. 
Working memory for manual gestures

Simons, D.J. \& Rensink, R.A. (2005). Change blindness: past, present, and future. Trends in Cognitive Sciences, 9(1), 16-20.

Smith, E. E. \& Jonides, J. (1997). Working memory: A view from neuroimaging. Cognitive Psychology, $33,5-42$.

Stanislaw, H \& Todorov, N (1999). Calculation of signal detection theory measures. Behavior Research Methods, Instruments, \& Computers, 31 (1): 137-149.

Sweet, L.H., Paskavitz, J.F., Haley, A.P., Gunstad, J.J., Mulligan, R.C., Nyalakanti, P.K., Cohena, R.A (2008). Imaging phonological similarity effects on verbal working memory. Neuropsychologia 46, $1114-1123$

Todd, J.J. \& Marois, R. (2004). Capacity limit of visual short-term memory in human posterior parietal cortex. Nature, 428(6984):751-4.

Wilson, M., \& Emmorey, K. (1997). A visuospatial "phonological loop" in working memory: Evidence from American Sign Language. Memory and Cognition, 25, 313-320.

Wilson, M. \& Fox, G. (2007). Working memory for language is not special: Evidence for an articulatory loop for novel stimuli. Psychonomic Bulletin \& Review, 14 (3), 470-473. 
Working memory for manual gestures

\section{Figure Captions}

Figure 1. Examples of partial presentation sequences for the n-back task. The grey squares indicate the quadrant of space in which the ball was caught in the particular items. " $\mathrm{S}$ " with index indicates item number. Targets in the particular task are marked "Trgt". The upper panels show examples with proximal foils for $n=1$ (left panel ) and $n=2$ (right panel). The lower panels show examples with distal foils only for $n=1$ (left panel ) and $n=2$ (right panel).

Figure 2. Significant interaction between load and foil proximity. Error bars show standard error. 
Table 1. Means and standard deviations (SD) for hits, errors, $d$ ' and response time (RT) on the $n$-back task

\begin{tabular}{|c|c|c|c|c|c|c|c|c|}
\hline & \multicolumn{4}{|c|}{$N=1$} & \multicolumn{4}{|c|}{$\mathrm{N}=2$} \\
\hline & \multicolumn{2}{|c|}{ Proximal } & \multicolumn{2}{|c|}{ Distal } & \multicolumn{2}{|c|}{ Proximal } & \multicolumn{2}{|c|}{ Distal } \\
\hline & Mean & SD & Mean & SD & Mean & SD & Mean & SD \\
\hline Hits & 14.00 & 1.08 & 14.05 & 1.00 & 12.85 & 1.50 & 12.05 & 1.64 \\
\hline Errors & 6.70 & 2.34 & 2.25 & 2.02 & 7.05 & 2.50 & 7.40 & 3.15 \\
\hline d' & 1.77 & 0.31 & 2.37 & 0.38 & 1.59 & 0.39 & 1.50 & 0.40 \\
\hline RT & 2601.74 & 323.65 & 2275.24 & 475.62 & 2767.46 & 354.29 & 2730.21 & 325.05 \\
\hline
\end{tabular}


Table 2. Spearman correlations between reading span, rated ball sense and performance on the $n$-back task

\begin{tabular}{lrcrc} 
& Reading span & \multicolumn{3}{c}{ Ball sense } \\
\cline { 2 - 5 } d' overall & Coefficient & $p$ & Coefficient & $p$ \\
d' $n=1$ & -0.301 & 0.197 & 0.480 & $0.0322^{*}$ \\
d' $\mathrm{n}=2$ & -0.001 & 0.997 & 0.530 & $0.016 *$ \\
Proximal & -0.342 & 0.140 & 0.317 & 0.174 \\
Distal & -0.256 & 0.275 & 0.521 & $0.019 *$ \\
& -0.292 & 0.212 & 0.248 & 0.291 \\
* indicates $\mathrm{p}<.05$ & & & &
\end{tabular}


gure / Figure

Click here to download high resolution image
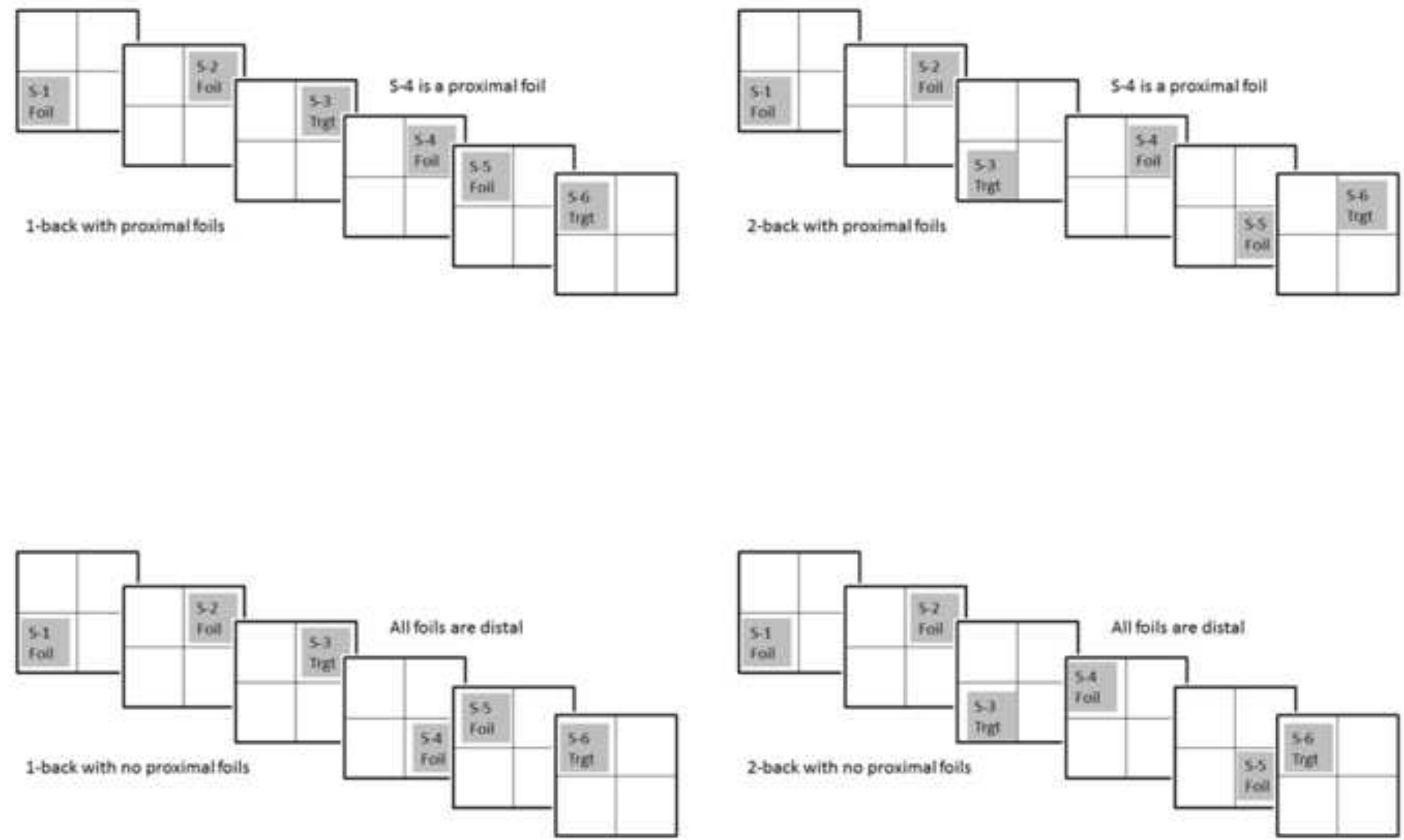


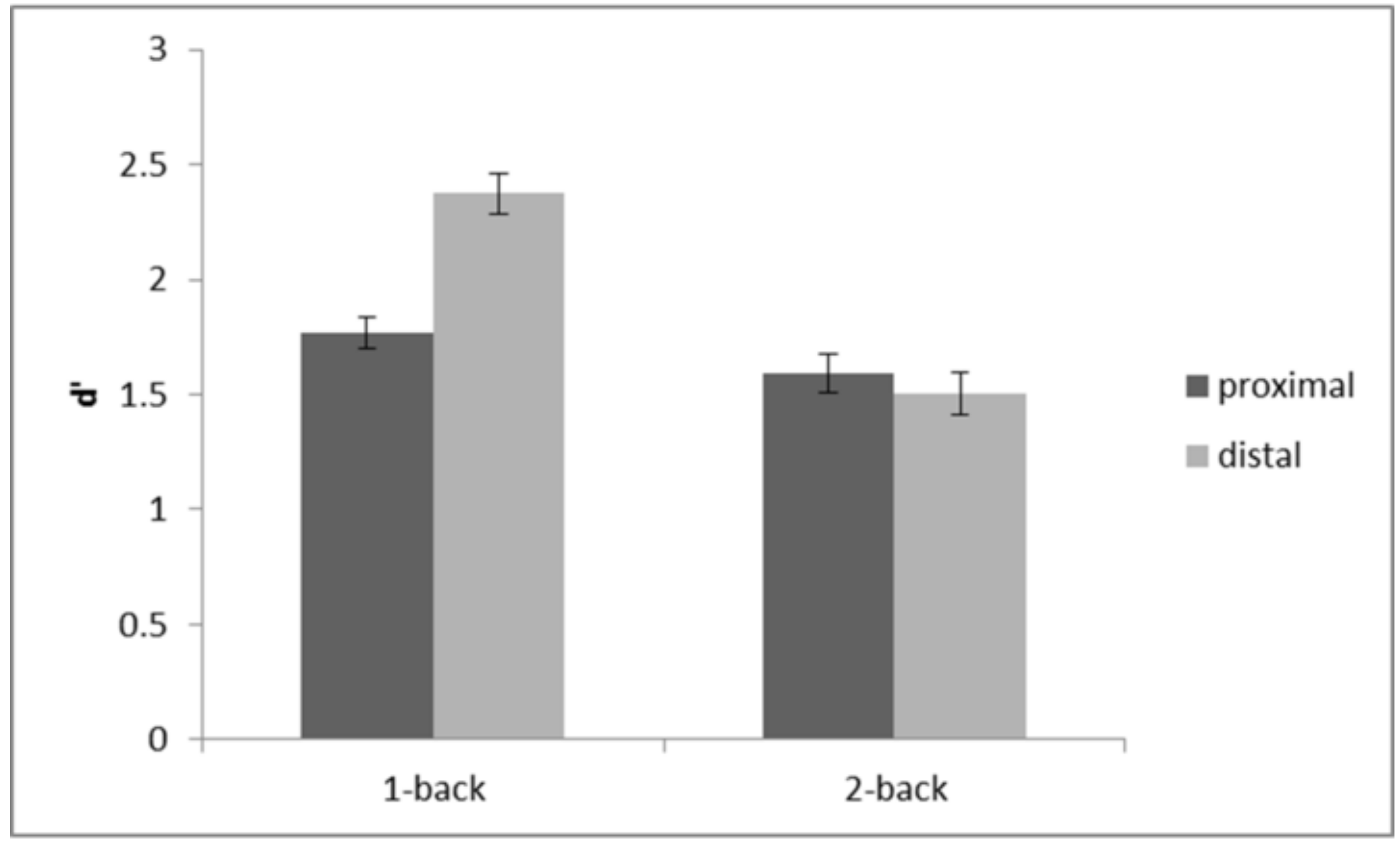

proximal distal

e
1-back 